\title{
RENDIMIENTOS EN DIFERENTES PROCESOS DE PULPAJE AL SULFITO
}

\section{ALDO ROLLERI (*), HERNÁN POBLETE (**) y ROBERTO JUACIDA (**)}

Instituto de Tecnología de Productos Forestales, Universidad Austral de Chile, casilla 567, Valdivia, Chile.

(*) Ingeniero Forestal.

(**) Doctor, Ingeniero Forestal.

\section{RESUMEN}

El objetivo general del estudio fue comparar los diferentes procesos de pulpaje al sulfito alcalino (AS, ASA, ASAM) como métodos de producción de pulpas de calidad papelera variando el factor $H$.

Para llevar a cabo el estudio, se utilizó astillas de Pinus radiata provenientes de un astillador industrial perteneciente al Instituto de Tecnología de Productos Forestales.

En cada uno de los procesos, las respuestas de pulpaje evaluadas fueron el rendimiento total clasificado, la cantidad de rechazo y la lignina residual.

Los resultados del estudio permiten confirmar que el poder reductor de la antraquinona favorece la delignificación y junto al metanol actúa como solvente de la lignina degradada, ayudando a disminuir los tiempos de cocción.

Del estudio se concluye que los tratamientos al sulfito alcalino (AS, ASA, ASAM) superan al testigo Kraft en el rendimiento total. No así en el rendimiento clasificado que presenta una situación inversa, en el que Kraft logra mayores valores de rendimiento.

No obstante, hay una tendencia de estos nuevos procesos de pulpaje de alcanzar los rendimientos clasificados de un Kraft industrial.

Palabras Clave: Pulpa, Proceso de pulpaje, Sulfito Alcalino; Procesos AS, ASA, ASAM.

\section{ABSTRACT}

General objective of this study was to compare different Sulfite Alkaline processes (AS, ASA, ASAM) as methods of pulp production changing the factor $H$.

As raw material Pinus radiata chips produced an industrial chipper were utilized.

The different processes were compared controling the unscreened yield, screened yield, screened rejects and residual lignin content.

The obteined values confirm the effect of Antraquinone aiding the delignification and, in a mixture with Methanol, helping to diminish the cooking time.

With the Sulfite Alkaline pulping methods (AS, ASA, ASAM) higher unscreened yield as with Kraft were obteined. 
The screened yield values obteined with the Alkaline sulfite pulping were lower as those of Kraft.

Keywords: Pulp, pulp process, Alkaline sulfite; AS, ASA, ASAM processes. 


\section{INTRODUCCIÓN}

El aumento progresivo de las normas que intentan proteger el medio ambiente; junto con el interés de las empresas de reducir sus costos energéticos, la alta inversión inicial y la emisión de compuestos azufrados altamente contaminantes del proceso Kraft, ha incentivado el desarrollo de otros procesos, como por ejemplo, los procesos al sulfito.

Una nueva generación de procesos al sulfito aparece en las últimas décadas con el uso de antraquinona y metanol.

Se desarrolla inicialmente el proceso denominado ASA (Sulfito Alcalino Antraquinona). La presencia del catalizador aumenta la velocidad de delignificación, y además, estabiliza las reacciones de degradación de los carbohidratos.

Paralelamente, se estudian los procesos denominados organosolv, en los que se utiliza alcoholes de bajo punto de ebullición (metanol). Este alcohol y la antraquinona poseen las ventajas de ser poco tóxicos y recuperables para su reutilización. El proceso identificado con la sigla ASAM (Sulfito alcalino, Antraquinona, Metanol), es el resultado final de investigaciones de procesos al sulfito y organosolv.

Este estudio está orientado a evaluar, a nivel de laboratorio, los resultados arrojados por tres tipos de procesos al sulfito alcalino: AS, ASA y ASAM; para luego compararlos entre sí y con proceso de pulpaje al sulfato, variando el factor $\mathrm{H}$, este método expresa los tiempos de cocción y temperatura como una variable única, variando el tiempo a temperatura máxima.

\section{OBJETIVOS}

El objetivo general del estudio es comparar los diferentes procesos al sulfito (AS, ASA, ASAM) como métodos de producción de pulpas de calidad papelera , variando el factor $\mathrm{H}$ :

Los objetivos específicos son evaluar las respuestas del proceso (AS, ASA, ASAM, Kraft), en función del rendimiento total, y clasificado de la pulpa; cantidad de rechazo, y contenido de lignina residual. 


\section{MATERIAL Y MÉTODO}

\section{Materia Prima}

Para los pulpajes se utilizaron astillas de Pinus radiata provenientes de un astillador industrial perteneciente al Instituto de Tecnología de Productos Forestales de la universidad Austral de Chile.

\section{Experiencia de Pulpajes}

Para llevar a cabo el estudio se realizaron pulpajes AS, ASA, ASAM y Kraft. De acuerdo con los objetivos del estudio, el diseño experimental que se aplicó para cada proceso al sulfito y el testigo Kraft, consideró como variable el factor $\mathrm{H}$. En total se realizaron 16 pulpajes, cuatro por cada proceso.

Las condiciones aplicadas se presentan en los Cuadros 1 al 4 .

\section{Cuadro 1}

\section{PROCESO AS (SULFITO ALCALINO)}

\begin{tabular}{|l|c|}
\hline \multicolumn{1}{|c|}{ Variable } & Condición \\
\hline Temperatura máxima $\left({ }^{\circ} \mathrm{C}\right)$ & 170 \\
\hline Carga de reactivo $(\%$ bms, óxido) & 25 \\
\hline Tiempo hasta temperatura máxima (min) & 90 \\
\hline Temperatura inicial $\left({ }^{\circ} \mathrm{C}\right)$ & 80 \\
\hline Factor $\mathrm{H}$ & $1.582 ; 2.044 ; 2.504: 2.965$ \\
\hline Relación Licor/madera & $4: 1$ \\
\hline Relación Sulfito/NaOH & $80 / 20$ \\
\hline
\end{tabular}

\section{Cuadro 2}

\section{PROCESO ASA (SULFITO ALCALINO, ANTRAQUINONA)}

\begin{tabular}{|l|c|}
\hline \multicolumn{1}{|c|}{ Variable } & Condición \\
\hline Temperatura máxima $\left({ }^{\circ} \mathrm{C}\right.$ ) & 170 \\
\hline Carga de reactivo $(\%$ bes, óxido) & 25 \\
\hline Tiempo hasta temperatura máxima (in) & 90 \\
\hline Temperatura inicial $\left({ }^{\circ} \mathrm{C}\right)$ & 80 \\
\hline Factor $\mathrm{H}$ & $1.582 ; 2.044 ; 2.504 ; 2.965$ \\
\hline Relación Licor/madera & $4: 1$ \\
\hline Antraquinona (\%bms) & 0,1 \\
\hline Relación Sulfito/ $/ \mathrm{NaOH}$ & $80 / 20$ \\
\hline
\end{tabular}


Cuadro 3

\section{PROCESO ASAM (SULFITO ALCALINO, ANTRAQUINONA, METANOL)}

\begin{tabular}{|l|c|}
\hline \multicolumn{1}{|c|}{ Variable } & Condición \\
\hline Temperatura máxima $\left({ }^{\circ} \mathrm{C}\right)$ & 170 \\
\hline Carga de reactivo $(\%$ bms, 6 xido) & 25 \\
\hline Tiempo hasta temperatura máxima (min) & 90 \\
\hline Temperatura inicial $\left({ }^{\circ} \mathrm{C}\right)$ & 80 \\
\hline Factor $\mathrm{H}$ & $1.582 ; 2.044 ; 2.504 ; 2.965$ \\
\hline Relación Licor/madera & $4: 1$ \\
\hline Antraquinona (\%bms) & 0,1 \\
\hline Metanol $(\%$ vol) & 25 \\
\hline Relación Sulfito/ $/ \mathrm{NaOH}$ & $80 / 20$ \\
\hline
\end{tabular}

Cuadro 4

PROCESO KRAFT (SULFATO ALCALINO)

\begin{tabular}{|l|c|}
\hline \multicolumn{1}{|c|}{ Variable } & Condición \\
\hline Temperatura máxima $\left({ }^{\circ} \mathrm{C}\right)$ & 170 \\
\hline Álcali activo como óxido & 17 \\
\hline Tiempo hasta temperatura máxima (min) & 90 \\
\hline Temperatura inicial $\left({ }^{\circ} \mathrm{C}\right)$ & 80 \\
\hline Factor $\mathrm{H}$ & $1.582 ; 2.044 ; 2.504 ; 2.965$ \\
\hline Relación Licor/madera & $4: 1$ \\
\hline Sulfidez $(\%)$ & 30 \\
\hline
\end{tabular}

Las respuestas por controlar en la pulpa AS, ASA, ASAM y Kraft fueron: rendimiento clasificado, rendimiento en rechazo y total, contenido de lignina remanente expresado como número Kappa (Tappi T $236 \mathrm{~cm}-85$ ).

Los resultados obtenidos del estudio planteado se sometieron a un análisis estadístico con el objeto de evaluarlos y posteriormente discutirlos.

\section{RESULTADOS Y DISCUSIÓN}

En la Figura 1 están expresados los rendimientos totales de AS, ASA, ASAM y el testigo Kraft. Se puede observar diferencias entre estos procesos.

El primer proceso por desarrollar fue AS que presenta un valor máximo de $77,2 \%$ con un factor $\mathrm{H}$ de 1.582. A medida que aumenta el tiempo a temperatura máxima, el rendimiento total disminuye por el aumento en las reacciones de delignificación, cayendo progresivamente hasta un $61 \%$ con un factor $\mathrm{H} 2.965$.

El alto rendimiento es atribuible a que el sulfito de sodio tiene baja velocidad de reacción, es un proceso comparable a la etapa química de un proceso semiquímico al 
sulfito neutro del cual se obtienen altos valores de rendimiento, particularmente dado por la estabilización de las hemicelulosas (KUTTUNEN, VIRKOLA Y YRJALA, 1979).

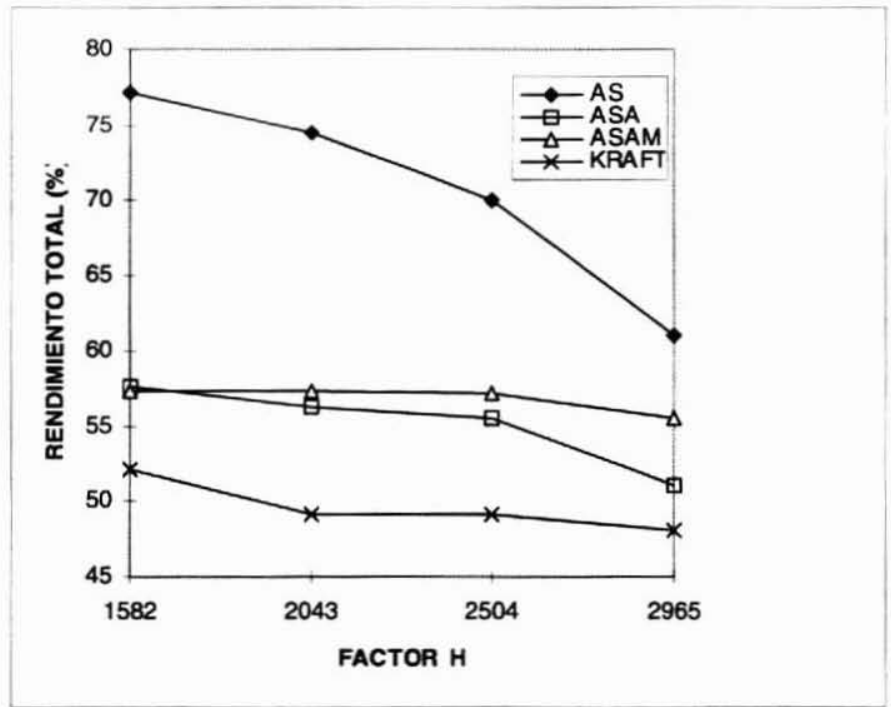

Figura 1. VALORES DE RENDIMIENTO TOTAL PARA LOS PROCESOS (AS, ASA, ASAM Y KRAFT), EN MADERA DE Pinus radiata

Se pudo determinar que AS necesita tiempo de cocción prolongados para obtener pulpas más finas. Si el material resultante fuera sometido a una etapa mecánica se obtendría una pulpa utilizable en la manufactura de papeles.

Al agregar antraquinona en el proceso ASA se logra acortar los tiempos de cocción. En la Figura 1, se puede observar que para obtener rendimientos entre 55\% y $65 \%$ BMS, AS requirió 180 minutos a $170^{\circ} \mathrm{C}$; en cambio ASA sólo ocupó 90 minutos a la misma temperatura para obtener igual rendimiento. Además se observa que ASA no sufre una variación muy pronunciada en todo el rango de factor $\mathrm{H}$, si se compara con AS, es decir, hay una tendencia a mantener el rendimiento de las pulpas a medida que avanza la delignificación se ve acentuada con la adición del metanol en el proceso ASAM.

En el caso de ASAM, se determinó que con un factor $\mathrm{H}$ de 1.582, el rendimiento total de $57,3 \%$ es muy parecido al presentado por ASA, no obstante, la variación es mínima para todo el rango de factor $\mathrm{H}$ cubierto, logrando una curva de pendiente casi constante, tendiendo a disminuir con el aumento a temperatura máxima. 
NAKANO, DAIMA, HOSOYA, e ISHIZO (1981) determinaron que ASAM es un proceso altamente selectivo en la delignificación, en el que el uso de metanol aumenta el grado de esta reacción, sin que el rendimiento sea afectado.

El proceso KRAFT presentó los valores de rendimiento total más bajo, $52,1 \%$ con un factor $\mathrm{H}$ de 1.582, para luego disminuir con el aumento de tal factor.

La comparación de procesos anteriormente descrita para el rendimiento total, es coincidente con el análisis de varianza aplicado. Se determinó que AS presenta diferencias significativas al compararlo con los demás procesos, en cambio ASA y ASAM, no presentaron diferencias significativas entre ellos.

Lo anterior confirma que estos procesos sean considerados como protectores de las hemicelulosas, es decir, que provocan una delignificación más selectiva, reflejando una tendencia más homogénea en los valores de rendimiento total en todo el rango de factor $\mathrm{H}$.

Las Figuras 2 y 3 muestran el rendimiento clasificado y el rechazo de los procesos de los procesos, respectivamente. En este análisis AS no está presente, ya que las pulpas obtenidas sin catalizadores están conformadas por un material con un bajo ataque químico y no pudieron ser desintegradas para lograr diferenciar entre clasificado y rechazo.

En la Figura 2 se presenta el rendimiento clasificado en función del tiempo a temperatura máxima. Se observa una situación inversa a la encontrada en el rendimiento total. ASA presenta el menor valor, $18,8 \%$ a un factor $\mathrm{H} 1.582$, para luego subir bruscamente hasta un $42,1 \%$ a factor $\mathrm{H} 2.965$. Esto se puede explicar al observar la Figura 3, en la que se presenta el rechazo en función del factor H. También se puede ver una tendencia idéntica, pero inversa al rendimiento clasificado; disminuye el rechazo a medida que aumentan los tiempos a temperatura máxima, es decir, al aumentar el factor $\mathrm{H}$, una parte del rechazo se convierte en clasificado.

En el caso de ASAM, se obtienen mayores valores de rendimiento clasificado y menor rechazo para un mismo factor $\mathrm{H}$, si se compara con ASA. Esta reacción es la esperada, dado que al efecto positivo de la antraquinona se suma el del metanol, que disminuye la tensión superficial y facilita la penetración de los reactivos, aumentando el rendimiento clasificado.

El proceso Kraft presenta la misma tendencia en los rendimientos clasificado y total, esto es que el porcentaje de rechazo es muy bajo, incluso cero.

En general, el rendimiento clasificado aumenta para todo el rango de factor $\mathrm{H}$. Si se compara estos resultados con los de ASA y ASAM, se observa una tendencia a acercarse a los rendimientos clasificados de Kraft. Lo anterior puede entenderse debido a que la acción de la antraquinona y el ,metanol se orienta a disminuir el porcentaje de rechazo, convirtiendo en clasificado, mediante una aceleración de la velocidad de delignificación por un macanismo redox (LÓPEZ y SOLÍS, 1989). 


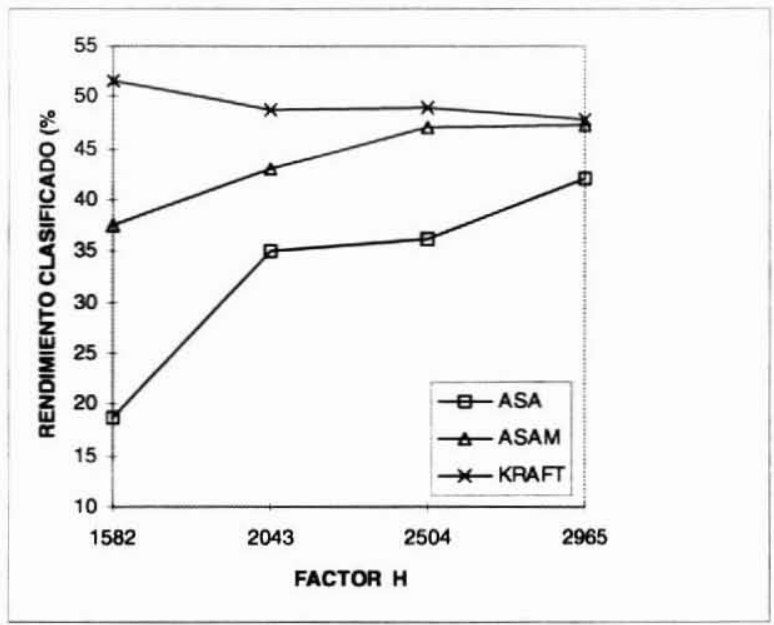

Figura 2. VALORES DE RENDIMIENTO CLASIFICADO PARA LOS PROCESOS (ASA, ASAM Y KRAFT), EN MADERA DE Pinus radiata.

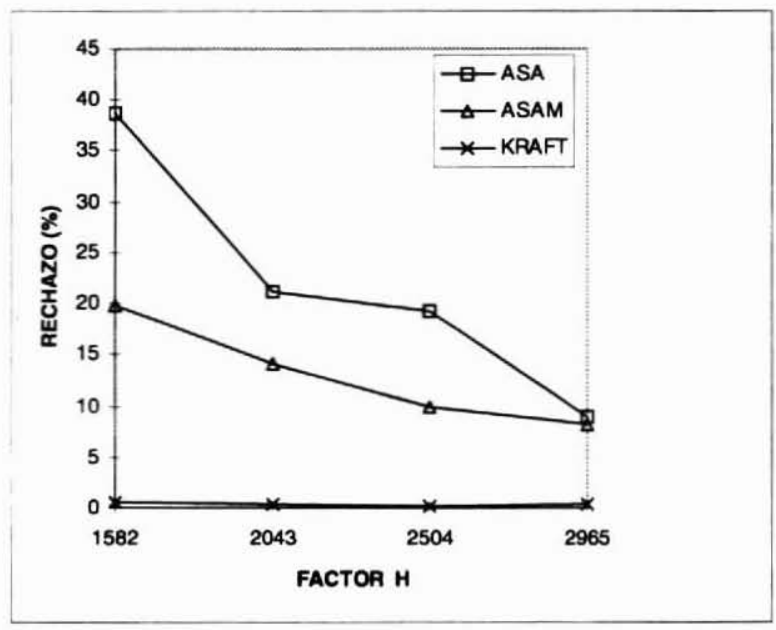

Figura 3. VALORES DE DE RECHAZO PARA LOS PROCESOS (ASA, ASAM Y KRAFT), EN MADERA DE Pinus radiata. 
En el análisis de varianza realizado para ASA, ASAM y Kraft hubo diferencias significativas al comparar los valores de rechazo. Como se puede ver en la Figura 3, ASA presenta el mayor valor, $38,7 \%$; seguido por ASAM y por último Kraft, en el que el rechazo llega a valores cercanos a cero.

Un análisis de varianza de todas las combinaciones posibles de los procesos en estudio, demostró que no hay una diferencia significativa entre ASA y ASAM, lo anterior se confirmó con el test de Duncan.

La homogeneidad encontrada no es bien expresada por la Figura 3. En ella Kraft presenta una diferencia clara con respecto a ASA y ASAM. Sin embargo, las diferencias de los promedios entre ASA y ASAM no dejan de ser importantes.

Como se menciona anteriormente, la tendencia de estos procesos es a acercarse a un pulpaje Kraft tipo, transformando el rechazo en rendimiento clasificado. ASAM logra este objetivo al estrechar la distancia de sus valores de rendimiento clasificado con los de Kraft; por ello no presentan diferencias significativas entre sí.

\section{Contenido de Lignina residual índice Kappa}

Los índices Kappa obtenidos en los diferentes procesos son presentados en la Figura 4. En ella se observa el índice Kappa en función de los tiempos a temperatura máxima, expresado por el factor $\mathrm{H}$.

La menor delignificación, debido a la poca reactibilidad de los compuestos del licor, la presenta el proceso AS.

Se puede apreciar que mayores tiempos de cocción no afectaron significativamente el contenido de lignina. Un factor $\mathrm{H}$ de 1.582 se registró un Kappa de 76,4, obteniéndose un valor final para este índice de 75,5 a un factor $\mathrm{H}$ de 2.965 .

Sin duda, este es un proceso con un rendimiento relativamente alto, que para ser utilizado en la manufactura de papeles, debería ser sometido a una fase mecánica inicial para cortar las fibras y, luego una fase química. Con eso se lograría una mayor separación de las fibras, similar a lo que ocurre en los procesos semiquímicos. 


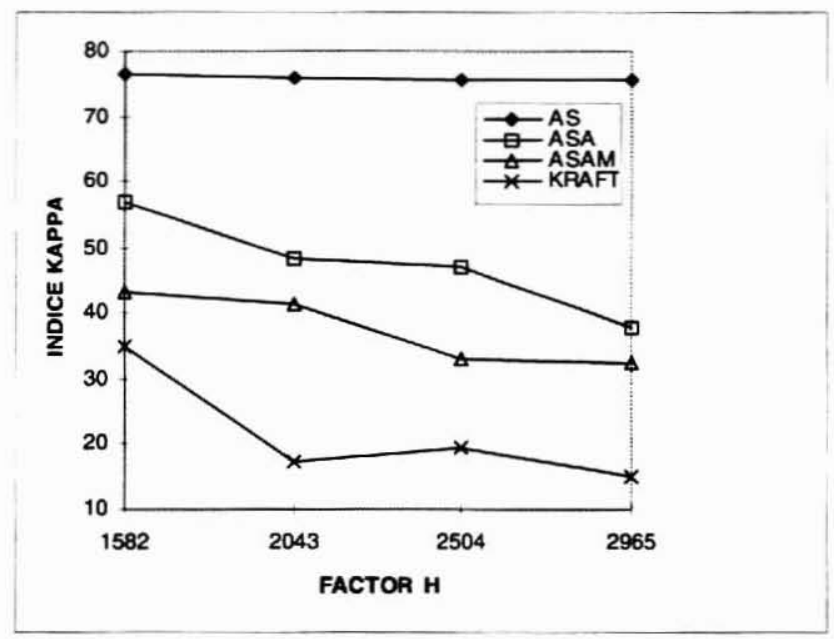

Figura 4. VALORES DE LIGNINA RESIDUAL PARA LOS PROCESOS (ASA, ASAM Y KRAFT), EN MADERA DE Pinus radiata.

En comparación al proceso AS, en el ASA la adición de antraquinona manifiesta su poder delignificador al disminuir el índice Kappa de 76,4 a 57,0, para un mismo factor $\mathrm{H}$ de 1.582. Lo anterior se traduce en una notable reducción de los tiempos de cocción. Esta baja en el índice Kappa entre As y ASA está ligada a los rendimientos totales anteriormente descritos.

Es importante tener presente que, a mayor delignificación, menor es el rendimiento de las pulpas. Se puede ver la fuerte variación dentro del mismo proceso ASA a medida que aumentan los valores del factor $\mathrm{H}$. Un índice Kappa de 57,0 a 1.582 en 19,1 puntos al bajar a un índice de 37,9 a un factor $\mathrm{H}$ de 2.965 .

La aplicación de un segundo catalizador para formar ASAM da origen a una nueva reducción del índice Kappa. A un factor $\mathrm{H}$ de 1.582, ASA presenta 57,0, en cambio ASAM logra 43,3. Además, la variación interna de este índice es ASAM es más pequeña que la presentada por ASA, y es igual a 1.076 puntos.

El metanol no tiene un poder de delignificación alto, pero sí es un solvente de la lignina residual (KORDSACHIA, WANDINGENN y PATT, 1993). Sumado esto a los efectos de la antraquinona, se origina una disminución más bien selectiva de la lignina, protegiendo los carbohidratos sin perjudicar los rendimientos. Lo anterior se puede observar en la Figura 1, de Rendimiento total respecto de factor $\mathrm{H}$, en que ASAM muestra rendimientos más altos que ASA. 
Hay una tendencia clara de estos procesos de alcanzar los resultados de un proceso Kraft industrial tipo, como el que se ve el la Figura 2. Debe tenerse presente que ASAM, es la última expresión de los procesos al sulfito que tiende a simular el comportamiento de un kraft, sin los perjuicios ecológicos de este último.

El análisis de varianza demostró que hay diferencias significativas en el contenido de lignina residual entre los procesos estudiados, que confirma el test de Duncan respectivo.

Esto es coincidente con los resultados logrados por KORDSACHIA, WANDINGER y PATT (1993). En ese trabajo se pueden ver claras diferencias en el índice Kappa, debido principalmente al efecto de antraquinona y metanol, aplicados como catalizadores.

Los autores citados utilizaron coníferas en sus trabajos, al igual que el presente estudio, ocuparon una relación $\mathrm{Na}_{2} \mathrm{SO}_{3} / \mathrm{NaOH}$ de $80 / 20$, antraquinona en un $0,1 \%$ y metanol $25 \%$ del volumen considerado para ASAM.

(Sin embargo, la variable temperatura máxima, considerada muy importante, no pudo ser desarrollada).

Los números Kappa 30-35, requeridos en la industria para obtener papeles finos, pueden ser obtenidos con el mismo tiempo de cocción con ASAM y Kraft. No obstante, los pulpajes ASAM requieren de temperaturas más altas, específicamente, sobre $180^{\circ} \mathrm{C}$ (ZIMMERMANN, PATT y KORDSACHIA, 1991). En la presente investigación sólo se alcanzó $170^{\circ} \mathrm{C}$, máxima capacidad de trabajo del digestor empleado.

En la Figura 4 se observa que ASAM alcanza un índice Kappa entre 30 y 35 a factores $\mathrm{H}$ de 2.504 y 2.965 . en tanto que con el proceso Kraft se requirió de un menor tiempo de cocción. Si se hubiera alcanzado los $180^{\circ} \mathrm{C}$, el aumento de la temperatura máxima de $10^{\circ} \mathrm{C}$, hubiera duplicado la velocidad de reacción (MELO y PAZ, 1972). Con este aumento de la temperatura máxima, se hubiera estrechado la diferencia entre los resultados de los procesos, siendo mayor el efecto entre ASAM y Kraft.

\section{CONCLUSIONES}

Los tres procesos al sulfito alcalino (AS, ASA y ASAM), superan al testigo Kraft en el rendimiento total.

Hay una tendencia a mantener el rendimiento total de las pulpas a medida que aumentan los tiempos a temperatura máxima expresados como factor $\mathrm{H}$ en los procesos ASA y ASAM.

Al comparar los tres procesos en un rango de rendimiento total de $55 \%$ a $65 \%$ bms, se observa que los catalizadores antraquinona y metanol logran acortar los tiempos de cocción. 
Las pulpas obtenidas mediante el proceso AS, son difíciles de desintegrar, ya que no alcanzan el punto de liberación de las fibras por lo que no pudo diferenciarse entre rendimiento clasificado y de rechazo.

En el rendimiento clasificado, el proceso al sulfato Kraft supera a los procesos al sulfito alcalino (ASAM y ASA), en todo el rango de factor $\mathrm{H}$ utilizado.

A medida que aumentan los tiempos a temperatura máxima, parte del rechazo originado en los procesos ASA y ASAM se convierte en clasificado.

Hay una tendencia de los procesos al sulfito alcalino (ASA y ASAM) de alcanzar los rendimientos clasificados del proceso kraft.

Los nuevos procesos al sulfito alcalino con la adición de catalizadores tienden a alcanzar los niveles de delignificación de un proceso Kraft.

El uso conjunto de antraquinona y metanol en el proceso ASAM, produce una nueva disminución en la lignina residual.

En el proceso ASAM se requieren menores tiempos de cocción que en cualquiera de os otros procesos al sulfito alcalino aplicados, para alcanzar un Kappa entre 30 y 40 .

Las diferencias alcanzadas en los valores de rendimiento y en los tiempos de cocción necesarios para lograrlos hubieran sido menores si se pudiese haber trabajado con temperaturas máximas similares.

\section{BIBLIOGRAFÍA}

Kettunen, J.; Virkola, N.E.; Yrjala, Y. 1979. The effect of antraquinone on Neutrasulphite and Alkaline Sulphite cooking of pine. Paperi ja Puu. 11: 685-694.

Kordsachia, O; Wandingenn, B and Patt, R. 1993. ASAM Pulping and Chlorine Free Bleaching Eucalyptus. Química \& Industria. Marzo $\mathrm{N}^{\circ} 1: 31-42$.

López, J.; Solís, O A. 1989. Pulpa de madera de Eucalyptus glabulus al sulfito alcalino en presencia de antraquinona y matanol. Universidad de Concepción. Cuartas Jornadas Técnicas de Celulosa y Papel 1991. Vol 1: $145-162$.

Melo, R.; Paz, J. 1972. Procesos semiquímicos de pulpaje. Programa Regional de Desarrollo Científico y Tecnológico. Proyecto Multinacional sobre Pulpa y Papel. universidad de Concepción, Laboratorio de Productos Forestales, Instituto de Investigación Tecnológica, Escuela de Ingeniería. 1972 2v.

Nakano, J.; Daima, H.; Hosaya, S. and Ishizu, A. 1981. Sulfite on Alkali - Methanol cooking. In: The Ekman Days 1981. Int Simp. wood pulp. chem, Stockolm, vol 2: 72 - 77.

Technical Association of the Pulp and Paper. 1989. Tappi Test Methods, Volume one - two.

Wong, A. 1980. Sulphite an option whose time has care again Tappi 63 (4): 53 - 57. 
Zimmeermann, M.; Patt, R.; Kordsachia, O. 1991. ASAM pulping of Douglas fir followed by a chlorine - free bleaching sequence. Tappi Journal. November: 129 - 134. 